\title{
A better spin on organic semiconductors
}

\author{
Progress in the field of organic spintronics has, to a degree, stagnated, but what can the community \\ do about it?
}

It is often the case that progress in a research topic that at first enjoyed a burst of initial discoveries begins to slow down. In such cases, it can be useful for the community to take a step back and to try and find the best way to move forward. This month we focus on the case of organic spintronics, which is perceived by parts of the community to be in need of a more structured approach.

Spin-dependent phenomena in organic semiconductors are different, and in some cases superior, from their inorganic counterparts. Organic semiconductors have weak spin-orbit coupling, resulting in long spin lifetimes, which are desirable for spin-based information storage and processing applications ${ }^{1,2}$. Furthermore, they are flexible and cheap to produce. All these properties have motivated efforts to develop spintronic devices that use organic materials, such as spin valves with organic spacers $^{3}$ and organic magnetic resonancebased magnetometers ${ }^{4}$.

\section{The field is not progressing}

\section{as swiftly as it could because}

of the recent proliferation

\section{of physical models of spin-} dependent properties that are tailored to specific data.

However, there is a downside to the properties of these organic materials: the weak spin-orbit coupling hinders the investigation of spin injection, manipulation and detection with the experimental and theoretical techniques routinely used for inorganic materials such as GaAs. Furthermore, organic semiconductors are highly disordered, resulting in hopping charge transport and low carrier mobility. These features makes it more difficult to study spindependent transport experimentally and to theoretically model spin- and magnetic field-related effects, such as organic magnetoresistance, which is the dependence of the resistance of an organic semiconductor on an applied magnetic field ${ }^{5}$. Finally, the low carrier mobility also limits applications to devices based primarily on localized spins ${ }^{2}$. As a result, there is some scepticism in the community that a technological outlet can ever be found for these materials, and clarifying issues on the fundamental properties of spin transport could help to shed light on the potential applications of organic spintronic devices.

In a Commentary in this issue, Christoph Boehme and John Lupton analyse the current fundamental understanding of spin processes in organic semiconductors ${ }^{6}$. In the authors' opinion, the field is not progressing as swiftly as it could because of the recent proliferation of physical models of spindependent properties that are tailored to specific data, rather than being conceived to describe general properties and then being scrutinized by experiment. This approach, they suggest, is causing confusion about the true origin of spin effects in organic semiconductors. They also discuss how recent experiments on organic magnetoresistance and measurements of spin-relaxation times have been questionably interpreted, by advancing conclusions on what model of spin transport best applies, without such conclusions being rigorously supported by the actual data.

Boehme and Lupton call on the community to reflect on the methods used, and return to a minimalistic approach to modelling, in which existing theories are rigorously scrutinized against measured data, before proposing new ad hoc models to explain given subsets of data. Moreover, they urge their peers to rely more heavily on the independent verification provided by spectroscopy techniques that can directly access spin states.

The Commentary focuses on a small number of case studies to exemplify common misconceptions that are currently affecting the field. The intention of the authors, as reiterated through correspondence with our editorial team, is to provide a general assessment of the attitude of the community, rather than criticise individual papers. They also do not aim to promote a particular model of spin transport over others, but to encourage the critical scrutiny of all proposed models against available experimental data.

\section{We welcome comments on}

the topic. We will aim to publish those Correspondence articles that we believe add constructively to the debate.

Before publication, we sent the Commentary to three researchers in the community, primarily to gauge the utility of such a discussion, as well as providing suggestions to clarify the message. Although these experts did not agree with every point made by Boehme and Lupton, they all acknowledged the potential usefulness of encouraging the community to reflect on the future of organic spintronics.

We hope that the publication of this Commentary will be the start of a discussion. As we did a year $\mathrm{ago}^{7}$, although in a different context, for the field of nanotoxicology, we welcome the submission of correspondence in the format of a letter to the editors. You may agree with the Commentary, but have different suggestions on how to proceed further, or you may in fact disagree with the authors' view; we welcome comments on the topic. We will aim to publish those Correspondence articles that we believe add constructively to the debate in our December issue. Please submit your contributions (maximum 600 words) through our online system or by e-mail to naturenano@nature.com (closing date: 4 October 2013).

\footnotetext{
References

1. Dediu, V. A., Hueso, L. E., Bergenti, I. \& Taliani, C. Nature Mater. 8, 707-716 (2009).

2. Szulczewski, G., Sanvito, S. \& Coey, M. Nature Mater. 8, 693-695 (2009).

3. Xiong, Z. H., Wu, D., Vardeny, Z. V. \& Shi, J. Nature 427, 821-824 (2004).

4. Baker, W. J. et al. Nature Commun. 3, 898 (2012).

5. Francis, T. L., Mermer, O., Veeraraghavan, G. \& Wohlgenannt, M New J. Phys. 6, 185 (2004).

6. Boheme, C. \& Lupton, J. Nature Nanotech. 8, 612-615 (2013).

7. Nature Nanotech. 7, 545 (2012).
} 